\title{
Sex Differences in Stress Response Circuitry Activation Dependent on Female Hormonal Cycle
}

\author{
Jill M. Goldstein, ${ }^{1,2,3,4,5,6}$ Matthew Jerram, ${ }^{1,2,3,4,6,7}$ Brandon Abbs, ${ }^{1,2,3,4,5}$ Susan Whitfield-Gabrieli, ${ }^{8}$ and Nikos Makris ${ }^{6,9,10,11}$ \\ Departments of ${ }^{1}$ Psychiatry and ${ }^{2}$ Medicine, ${ }^{3}$ Division of Women's Health, and ${ }^{4}$ Connors Center for Women's Health and Gender Biology, Brigham and \\ Women's Hospital and Harvard Medical School, Boston, Massachusetts 02120, ${ }^{5}$ Division of Psychiatric Neuroscience, Department of Psychiatry, \\ Massachusetts General Hospital and Harvard Medical School, Charlestown, Massachusetts 02129, ${ }^{6}$ Athinoula Martinos Center for Biomedical Imaging, \\ Massachusetts General Hospital and Massachusetts Institute of Technology, Charlestown, Massachusetts 02129, ${ }^{7}$ Department of Psychology, Suffolk \\ University, Boston, Massachusetts 02108, ${ }^{8}$ Department of Brain and Cognitive Sciences, Massachusetts Institute of Technology, Cambridge, Massachusetts \\ 02139, and Departments of ${ }^{9}$ Psychiatry and ${ }^{10}$ Neurology and ${ }^{11}$ Center for Morphometric Analysis, Massachusetts General Hospital and Harvard Medical \\ School, Charlestown, Massachusetts 02129
}

Understanding sex differences in stress regulation has important implications for understanding basic physiological differences in the male and female brain and their impact on vulnerability to sex differences in chronic medical disorders associated with stress response circuitry. In this functional magnetic resonance imaging study, we demonstrated that significant sex differences in brain activity in stress response circuitry were dependent on women's menstrual cycle phase. Twelve healthy Caucasian premenopausal women were compared to a group of healthy men from the same population, based on age, ethnicity, education, and right handedness. Subjects were scanned using negative valence/high arousal versus neutral visual stimuli that we demonstrated activated stress response circuitry [amygdala, hypothalamus, hippocampus, brainstem, orbitofrontal cortex (OFC), medial prefrontal cortex (mPFC), and anterior cingulate gyrus (ACG)]. Women were scanned twice based on normal variation in menstrual cycle hormones [i.e., early follicular (EF) compared with late follicular-midcycle (LF/MC) menstrual phases]. Using SPM8b, there were few significant differences in blood oxygenation leveldependent (BOLD) signal changes in men compared to EF women, except ventromedial nucleus (VMN), lateral hypothalamic area (LHA), left amygdala, and ACG. In contrast, men exhibited significantly greater BOLD signal changes compared to LF/MC women on bilateral ACG and OFC, mPFC, LHA, VMN, hippocampus, and periaqueductal gray, with largest effect sizes in mPFC and OFC. Findings suggest that sex differences in stress response circuitry are hormonally regulated via the impact of subcortical brain activity on the cortical control of arousal, and demonstrate that females have been endowed with a natural hormonal capacity to regulate the stress response that differs from males.

\section{Introduction}

Although animal studies support the notion that hormonal differences contribute to explaining sex differences in stress response circuitry, no one has directly tested this in the human brain. This has widespread implications for clinical medicine, given that response to stress has been implicated in the vulnerability for numerous disorders with known sex differences in incidence, such as depression and anxiety disorders, cardiovascular disease, and other chronic medical disorders. Further, an understanding of sex differences in the regulation of stress has important

\footnotetext{
Received June 25, 2009; revised 0ct. 14, 2009; accepted Nov. 5, 2009.

This work was supported by grants from the National Institute of Mental Health to J.M.G. (NIMH-ORWH P50 MH082679, NIMH R01 MH56956) and the Athinoula Martinos (enter for Biomedical Imaging at Massachusetts General Hospital Collaborative Start-up Award. We also thank Dr. Bernard Harlow for access to the female population sample, Dr. Russell Poldrack for statistical help on the design of earlier versions of this study, Dr. Deborah Walder and Gabe Daly for help on some earlier stages of data analyses, Sarah Spaeth for help with data acquisition and analysis, George Papadimitriou for help in image production, Drs. Anne Klibanski and Stuart Tobet for comments on earlier aspects of the study, and Lisa Cushman-Daly for manuscript preparation.

Correspondence should be addressed to Dr. Jill M. Goldstein, Division of Women's Health, Brigham and Women's Hospital, One Brigham Circle, 1620 Tremont Street, Boston, MA 02120. E-mail: jill_goldstein@hms.harvard.edu. D0I:10.1523/JNEUROSCI.3021-09.2010

Copyright $\odot 2010$ the authors $\quad 0270-6474 / 10 / 300431-08 \$ 15.00 / 0$
}

implications for understanding basic physiological differences in the male and female brain.

Brain regions in stress response circuitry include subcortical regions, central amygdala (AMYG), hypothalamus [paraventricular nucleus (PVN), ventromedial nucleus (VMN), and lateral hypothalamic area (LHA)], hippocampus, and brainstem [e.g., periaqueductal gray (PAG)], and cortical regions, orbitofrontal cortex (OFC), medial prefrontal cortex (mPFC), and anterior cingulate gyrus (ACG), referred to as playing a role in the cortical control of arousal (Keverne, 1988; McEwen and Magarinos, 1997; Price, 1999). These regions are sexually dimorphic in animal and human studies and regulate the hypothalamic-pituitary-adrenal (HPA) and hypothalamic-pituitary-gonadal (HPG) axes (Keverne, 1988; Tobet and Hanna, 1997; Goldstein et al., 2001; Ostlund et al., 2003; Swaab, 2004; Bao et al., 2005), suggesting hormonal regulation of stress response circuitry.

Functional brain imaging studies provide a unique opportunity to test the impact of hormones on explaining sex differences in this circuitry in in vivo human paradigms. Behavioral and imaging studies with negative/high arousal stimuli have demonstrated significantly increased brain activations in men and women 
in stress response regions. Although inconsistent (Schienle et al., 2005), women demonstrated greater arousal to aversive cues than men (Lane et al., 1997; Lang et al., 1998; Bradley et al., 2001; Caseras et al., 2007), and greater signal intensity changes in AMYG, ACG, and/or OFC (Wrase et al., 2003; McClure et al., 2004), even when both sexes had similar subjective stimuli ratings (McClure et al., 2004). However, no imaging studies have directly tested the full circuitry or explanatory models for sex differences in the stress response.

We previously demonstrated, in a functional magnetic resonance imaging (fMRI) paradigm of aversive arousal in healthy women scanned twice, increased arousal and brain activity in stress response circuitry during the early follicular (EF) menstrual phase (when estrogen and progesterone were low), compared with attenuated brain activity during late follicular/midcycle phase (LF/MC) (when estrogen was high and progesterone still relatively low), which was attributed to attenuating effects of estrogen (Goldstein et al., 2005). The purpose of the current study was to test whether hormonal effects on activations of the stress response circuitry in the women explained sex differences in activations of this circuitry. We predicted that men would look more similar to women in EF than during LF/MC, given the greater similarity of men's hormonal status to women's hormonal status at EF compared to LF/MC. In this study, we demonstrated that females are endowed with a natural hormonal capacity to regulate the stress response that differs from males, and suggest that these hormones act through the impact of subcortical brain activity on the cortical control of the stress response.

\section{Materials and Methods}

\section{Subjects}

Participants in the present study were selected from a community sample of women drawn by the Harvard Mood and Cycles Study (Harlow et al., 1999 ) and men from the same geographic area. Subjects were aged 3546, right handed, and Caucasian, had at least high school education, English as their first language, and no current psychiatric disorder, and were free of Axis I psychiatric disorders during their lifetime. The exclusion criteria also required absence of the following: (1) substance abuse within the last 6 months; (2) history of head injury with any documented cognitive sequelae or loss of consciousness for $>5 \mathrm{~min}$; (3) neurologic disease; (4) mental retardation; and (5) medical illness that may significantly impair neurocognitive function. Further, women were required to have regular menstrual cycles for at least 1 year before inclusion in the present study. They knew their cycles well, given that they had kept daily diaries of their cycles in the Harvard Moods and Cycles study for $\sim 5$ years charting their menstrual cycles, validated by blood assays obtained regularly (Harlow et al., 1999).

Twelve right-handed Caucasian women were systematically ascertained from the above community population study, and reported in a previous analysis by Goldstein et al. (2005). All participants were unmedicated and free of medical, neurological, and psychiatric illness and sensory impairments, had regular monthly cycles greater for $>1$ year, and reported no changes in the description of the flow of their menstrual cycles over the last year as well as other cycle characteristics, such as pain or menstrual discomfort. All gave informed consent and received an honorarium for participation. The mean age for the women was 43.9 years ( $\mathrm{SD}=1.4$ years), ranging from 41 to 46 years old. All women were normally cycling, and no one was on oral contraception.

With regard to the number of weeks between scans, all women were scanned within a 2 week interval between the beginning of their menstrual cycle and midcycle, even for those women whose first visit was not the beginning of her cycle. The range of menstrual cycle days on which women were scanned were days $2-14$ of their menstrual cycles. There was a median menstrual cycle time of "day $3 \pm 2$ d" (representing early follicular, when scanning occurred on days 2-5) and "day $12 \pm 2 \mathrm{~d}$ " (representing midcycle, when scanning was conduced on menstrual cycle days 10-14). We understand, given the lack of blood assays at the time of scanning, that some women, particularly the older women, may have been in the luteal phase rather than in midcycle, given the shorter follicular phase of some older women. However, all women had charted their cycles for 3 months before scanning to provide accurate cycle timing by self-report, given that there was a 1 year period between the daily diaries of their cycle obtained in the study by Harlow et al. (1999) and the scanning in our study. The range of time between scans in days was $10-14 \mathrm{~d}$

In the current study, 13 men were systematically sampled from the same geographic area as the community sample of women and made comparable on age, ethnicity, education, and right handedness. The mean age of the men was 39.1 years $(S D=2.3)$, ranging from 35 to 43 years old. There were no significant differences between the age of the men and women and they were comparable on education, i.e., all had at least a high school education. Adjusting for age in our general linear models did not affect the results presented here.

\section{Clinical procedures}

Before and after each scan, each participant was administered the Profile of Mood States (POMS) and the Spielberger State-Trait Anxiety Inventory (STAI). Participants were asked to rate their current mood. The POMS is an adjective checklist of 72 items, rated by the participant on a scale from 0 to $4(0=$ does not apply; $4=$ the adjective describes emotional state extremely well). Scales include Anxiety, Vigour, Fatigue, Confusion, Anger, and Depression, out of which an overall mood state score is derived as well.

The Spielberger State-Trait Anxiety Inventory is a questionnaire designed to assess an individual's perception of current and "usual" levels of anxiety. The individual rates 40 statements regarding feelings of anxiety on a scale from 1 to $4(1=$ statement poorly reflects feelings of anxiety; $4=$ statement accurately reflects feelings of anxiety). Statements reflect how the individual feels in general (reflecting trait-level anxiety) and current feelings of anxiety (reflecting state-level anxiety), out of which an overall rating is also calculated. The STAI was administered before and after scanning to assess state- and trait-level anxiety and to test for similarities across the two menstrual cycle phases. All interviews were performed by one of the authors (M.J.). Overall POMS and STAI scores (presented in Table 1) have been standardized to clinical populations. Any score $<50$ is in the low normative range. Thus, as seen in Table 1, all subjects were similar in their clinical ratings and were in the low/normal range of anxiety or mood-related symptoms (Table 1). Further, there were no significant differences found in the change in mood state prescan to postscan, indicating that the stimuli had similar effects on all groups. Thus, controlling for mood state did not change the results.

\section{Stress response task}

Stimulus materials were drawn from the International Affective Picture System (IAPS) (Lang et al., 2008), a system of color pictures that were rated by large groups of subjects along the dimensions of affective valence (pleasant-unpleasant) and arousal (calm-aroused). Pictures were systematically drawn from the set according to affective valence (unpleasant and neutral) and arousal (high and low) based on normative ratings developed by Lang et al. (1993). Two sets of pictures, each containing 72 images, were selected-one of unpleasant valence and high arousal and the other of neutral valence and low arousal. Faces and scenes were counterbalanced between high arousal and neutral slides. To create the 72 fixation slides, the neutral valence/low arousal slides were transformed using Fourier transforms to create a set of slides with the same physical properties of the original but without content that was readily recognizable.

During the functional scans, participants were presented with three blocks of stimuli in a counterbalanced method. One block of stimuli consisted of fixation slides presented at the rate of one every five seconds for $30 \mathrm{~s}$. The other two blocks consisted of six arousing negative affect images and six neutral in valence and low arousal. Six different pictures were presented for five seconds in each block, which lasted $30 \mathrm{~s}$, with blocks repeated four times during each 6 min functional scan. Three 
Table 1. Behavioral measures of mood and anxiety before and after scanning

\begin{tabular}{|c|c|c|c|}
\hline & $\begin{array}{l}\text { Men } \\
\text { mean (SD) }\end{array}$ & $\begin{array}{l}\text { EF women } \\
\text { mean (SD) }\end{array}$ & $\begin{array}{l}\text { LF/MC womer } \\
\text { mean (SD) }\end{array}$ \\
\hline \multicolumn{4}{|l|}{ Mood scale $^{a}$} \\
\hline \multicolumn{4}{|l|}{ Anxiety } \\
\hline Prescan & $31.5(3.2)$ & $28.2(3.8)$ & $27.9(2.8)$ \\
\hline Postscan & $30.3(0.7)$ & $28.8(3.6)$ & $28.8(3.3)$ \\
\hline \multicolumn{4}{|l|}{ Depression } \\
\hline Prescan & 36.7 (3.6) & $33.0(2.3)$ & $32.7(1.6)$ \\
\hline Postscan & $35.6(1.0)$ & $32.7(1.4)$ & $32.4(0.9)$ \\
\hline \multicolumn{4}{|l|}{ Anger } \\
\hline Prescan & $39.2(3.4)$ & $37.6(1.6)$ & $38.3(2.3)$ \\
\hline Postscan & $37.2(0.4)$ & $37.1(0.3)$ & $37.1(0.3)$ \\
\hline \multicolumn{4}{|l|}{ Vigor } \\
\hline Prescan & $65.3(13.7)$ & $68.2(11.0)$ & $68.3(15.0)$ \\
\hline Postscan & $63.6(15.5)$ & $65.6(11.7)$ & $66.8(16.6)$ \\
\hline \multicolumn{4}{|l|}{ Fatigue } \\
\hline Prescan & $43.3(6.7)$ & $39.0(5.0)$ & $37.9(4.4)$ \\
\hline Postscan & $40.5(5.4)$ & $39.6(6.1)$ & $37.1(4.7)$ \\
\hline \multicolumn{4}{|l|}{ Confusion } \\
\hline Prescan & $32.3(0.9)$ & $30.4(0.8)$ & $30.2(0.6)$ \\
\hline Postscan & $32.4(1.0)$ & $30.0(1.0)$ & $30.9(1.8)$ \\
\hline \multicolumn{4}{|l|}{ Anxiety scale $^{b}$} \\
\hline Trait anxiety & $25.3(2.9)$ & $27.3(7.9)$ & $26.9(7.0)$ \\
\hline \multicolumn{4}{|l|}{ State anxiety } \\
\hline Prescan & $29.7(7.6)$ & $25.9(4.9)$ & $26.9(5.3)$ \\
\hline Postscan & $27.2(6.1)$ & $28.0(6.2)$ & $26.4(5.5)$ \\
\hline
\end{tabular}

Scores presented are $t$ scores. There were no significant differences between any of the above groups on clinica ratings. All were in the low-level, normative range.

${ }^{a}$ POMS rates current mood (self-report); it consists of 72 items, rated on a scale from 0 to 4 ( $0=$ does not apply; $4=$ the adjective describes emotional state extremely well). The table reflects a standardized overall mood state score; any score $<50$ is in the low, normative range.

${ }^{b}$ STAl is a self-report of current and "usual" levels of anxiety. Forty statements are rated on a scale from 1 to 4 ( $1=$ statement poorly reflects feelings of anxiety; $4=$ statement accurately reflects feelings of anxiety). Statements reflect how the individual feels in general (reflecting trait-level anxiety) and current feelings of anxiety (reflecting state-level anxiety), out of which a standardized overall rating is also calculated. Any score $<50$ is in the low, normative range.

functional scans were taken, resulting in 12 blocks of each condition. In all conditions, the participant was asked to press the button under her or his index finger each time the picture being presented changed to ensure attention to the presented stimuli.

\section{Experimental setup and procedure}

The women were brought in for two sessions, during early follicular menstrual cycle phase (at onset of menstrual cycle) and during midcycle. In a counterbalanced design, half of the women came in for their first session within $2-3 \mathrm{~d}$ of their predicted date of ovulation. This group then returned for the second session, all within 2 weeks after their first visit. The other group came in for their first session within 2 weeks after their predicted date of ovulation and then returned $\sim 2$ weeks after that first visit. Counterbalancing visit with menstrual cycle phase controlled for potential confounding effects of "time" and/or "novelty." The men were compared to these women at two different points in their menstrual cycle.

On arrival, the study was explained in detail, informed consent was obtained, and participants were administered the mood measures. After entry into the magnet room, participants were given the arousal task instructions and fit with earplugs to attenuate scanner noise. Participants lay prone on the scanner gurney and foam padding was placed across the forehead to prevent head motion. Physiological monitoring sensors were placed on the fingers of the left hand of the participant and the participant was given a response box with two buttons for the right hand. The head coil was then placed over the head and the participant placed into the scanner.

A Macintosh G4 Computer (Apple Computer) running MacStim experimental presentation program (WhiteAnt Occasional Publishing) was used to produce the visual stimuli. Visual stimuli were presented via an liquid crystal display projector through a custom lens onto a screen situated in the magnet bore. These stimuli were viewed through a mirror attached to the head coil. The computer was in the control room outside the Faraday shield of the scanner. Stimuli were presented as described previously. When scanning was completed, the participant was again administered the POMS and state portion of the STAI. This procedure was repeated during the second visit.

\section{Imaging}

Scanning was performed with a quadrature full head coil and a $1.5 \mathrm{~T}$ Sonata MR scanner (Siemens) modified for echo-planar imaging. A single slice two-dimensional spoiled gradient recall (SPGR) axial localizer scan was acquired, followed by a 60 slice sagittal localizer scan [conventional T1-weighted magnetization-prepared rapid-acquisition gradient echo (MPRAGE) sequence; field of view $(F O V)=23 \times 17.25 \mathrm{~cm}$; matrix $=256 \times 192$; in-plane resolution $=0.90 \mathrm{~mm}$; slice thickness $=2.8$ $\mathrm{mm}$ ] to orient; for subsequent scans, 15 contiguous axial slices along the anterior commissure-posterior commissure (AC-PC) line and covering the whole brain. This was followed by an automated shim procedure to improve $\mathrm{B}_{0}$ field homogeneity and a MP-RAGE T1-weighted flowcompensated scan $(\mathrm{FOV}=40 \times 20 \mathrm{~cm}$; matrix $=512 \times 256$; in-plane resolution $=0.78 \mathrm{~mm}$; slice thickness $=7 \mathrm{~mm} ; 15$ slices coplanar with the functional slices). The next scan was a 15-slice T1-weighted echo planar inversion recovery sequence [repetition time (TR) $=20 \mathrm{~s}$; echo time $(\mathrm{TE})=40 \mathrm{~ms}$; inversion time $(\mathrm{TI})=1100 \mathrm{~ms}$ FOV $=40 \times 20 \mathrm{~cm}$; matrix $=256 \times 128$; in-plane resolution $=1.57 \mathrm{~mm}$; slice thickness $=7$ $\mathrm{mm}$ ] coplanar to the functional images for anatomic localization. Finally, a series of functional scans were acquired, using an asymmetric spin echo, $\mathrm{T} 2 *$-weighted sequence $(\mathrm{TR}=2000 \mathrm{~ms}$; $\mathrm{TE}=70 \mathrm{~ms}$; refocusing pulse offset by $-25 \mathrm{~ms} ; 1$ excitation; FOV $=40 \times 20 \mathrm{~cm}$; matrix $=$ $128 \times 64$; in-plane resolution $=3.125 \mathrm{~mm}$; slice thickness $=7 \mathrm{~mm} ; 15$ contiguous slices along the AC-PC line). This pulse sequence has excellent sensitivity to parenchymal signal changes concurrent with experimental perturbation, and reduced macrovascular sensitivity. Functional scans were acquired for 184 time points per experimental run (4 time points of scanner shimming and 180 time points of A-B-A-B blocks). Three sets of functional scans were acquired.

\section{Data analysis}

fMRI data were preprocessed using Statistical Parametric Mapping (SPM8b) (Wellcome Department of Cognitive Neurology, 2008) and using custom routines in MATLAB (The MathWorks, 2000). Preprocessing included correction for acquisition timing across slices, bulk-head motion, and spatial smoothing with a Gaussian filter $(8 \mathrm{~mm}$ at full-width at half-maximum). No individual runs exhibited head motion $>2.8 \mathrm{~mm}$ across all runs. After motion correction and spatial smoothing, images for each subject were spatially normalized using nonlinear volume-based spatial normalization techniques within SPM. The template used by SPM is the standard brain template developed at the Montreal Neurological Institute (MNI). Following preprocessing, statistical analysis was performed at the single-subject level using SPM. SPM treats each voxel's blood oxygenation level-dependent (BOLD) time series according to a general linear model. As this was a block design, each epoch of trials was modeled using a boxcar function convolved with a canonical hemodynamic response function. Low-frequency components of the fMRI signal were modeled as confounding covariates using a set of cosine basis functions to increase sensitivity to signals of interest. Specific comparisons of interest (negative valence/high arousal versus neutral valence/low arousal) were tested using linear contrasts, and SPM maps were created based on these contrasts. These contrast values (which represent estimates of the mean signal change at each voxel) were used in statistical analyses.

Voxelwise analyses. Results from the individual subject level were submitted to a second level analysis in which subjects were treated as a random effect. For a single group, activation was assessed using a onesample $t$ test to compare the value of the contrast images against zero. For multiple groups, independent-sample $t$ tests were used to compare the size of a particular effect between groups. To correct for multiple comparisons on the voxelwise error rate, we adopted methods implemented by SPM that employ the theory of Gaussian random fields (Friston et al., 
2004) to obtain statistical maps with a single $\alpha$ level across the search volume of interest. Given that we had specific hypotheses about a set number of brain regions, we used the approach in SPM8b referred to as small volume correction (SVC), which limits voxelwise analyses to voxels within our a priori regions of interest. Anatomically defined regions of interest included the amygdala, hippocampus, hypothalamus, midbrain regions, OFC, ventromedial prefrontal cortex (vmPFC), and ACG. False positives were controlled using a combination of a voxelwise height threshold ( $p<0.005$ uncorrected) and an extent threshold, that jointly resulted in a cluster-level false-positive level of $p<0.05$, corrected for multiple comparisons within the search volume using familywise error (FWE) correction based on random field theory. The FWE rate is a more conservative correction for multiple comparisons within fMRI data. Anatomic borders were defined using overlays created using the Wake Forest University (WFU) Pickatlas (Maldjian et al., 2003) toolbox for SPM to match the borders of the hypothesized regions on the canonical brain provided in SPM8b. Using the SVC tool in SPM, activations within the borders of the overlays were identified.

Functional region of interest definition. Functional regions of interest (ROIs) were defined from the main effect (across both sexes and both female time points) of the negative - neutral contrast. The statistical maps of the negative $>$ neutral condition, including by menstrual phase, were examined at the uncorrected $p<0.01$ level. Effect sizes were reported for all activations within our ROIs (see below). Activated clusters within the ROIs were labeled by visual inspection of our neuroanatomist (N.M.) and comparison to the MNI neuroanatomical atlas. The neuroanatomist (N.M.) was blind to study hypotheses and specific nuclei of interest before identification of the significant BOLD signal intensity differences in activation, thus underscoring the validity of the neural network activated by our stimuli.

Functional ROI analyses. After identifying clusters within the ROIs, WFU Pickatlas was used to build spherical overlays with the center of the sphere at the SPM-identified peak of activation within each cluster. These overlays were then used on the statistical maps of each individual to acquire signal change values across a specific region of interest, which was then used in general models for later statistical comparisons. Values indicate the degree of change in MR signal detected between the negative arousal condition and neutral condition and are expressed in terms of percentage signal change. Average effect sizes (percentage BOLD signal change averaged across all voxels within a sphere) comparing the negative versus neutral conditions were obtained for each ROI using the REX toolbox for SPM8b. If more than one cluster was found within a single anatomical region of interest, the signal change values for all spheres within the ROI were averaged. The effect sizes combine voxel-level data across an entire region of interest (see Table 3 results), regardless of whether the change in signal for that voxel passes the voxel-level threshold (reported in Table 2). Thus, the effect sizes are "more representative" of signal intensity values within an anatomically identified region of interest, as they represent an analysis of a priori anatomic regions of interest that do not rely only on voxel- or cluster-level significance.

The formula for calculating these effect sizes (ESs) is as follows: ES = (negative condition signal change - neutral condition signal change)/SD of signal intensity value of the whole sample across all conditions. The effect sizes (SD units) were then compared between the three groups (men, EF women, and LF/MC women) using PROC MIXED in SAS. This procedure fits various mixed linear models and can appropriately handle repeated measurements, a function that is necessary to take into account the variance of the two different measurements, EF and LF/MC, for each woman in the current study.

\section{Results}

Comparisons of men versus EF women and men versus LF/MC women are shown in Table 2 and Figure 1. Negative-to-neutralstimuli in men compared to EF women showed few significant sex differences in our hypothesized ROIs, with none significant at a cluster level FWE-corrected $p<0.05$ level (Table 2). There were no significant differences in comparisons of EF women versus men.
Table 2. Regions of activation in comparisons of negative valence to neutral valence conditions: men compared with women in the early follicular and midcycle menstrual phases

\begin{tabular}{|c|c|c|c|c|c|c|c|}
\hline \multicolumn{2}{|l|}{ Hypothesized regions } & \multirow[b]{2}{*}{$x$} & \multirow[b]{2}{*}{$y$} & \multirow[b]{2}{*}{$z$} & \multirow[b]{2}{*}{$Z$} & \multirow[b]{2}{*}{ Voxels } & \multirow{2}{*}{$\begin{array}{l}\text { FWE }^{a} \\
p \text { value }\end{array}$} \\
\hline Region & Hemisphere & & & & & & \\
\hline \multicolumn{8}{|c|}{ Early follicular (men > women) } \\
\hline Amygdala & $\mathrm{L}$ & -18 & -4 & -14 & 2.92 & 27 & 0.32 \\
\hline Amygdala & $\mathrm{R}$ & 20 & 0 & 14 & 2.74 & 18 & 0.41 \\
\hline \multicolumn{8}{|l|}{$A C G$} \\
\hline BA 32 & $\mathrm{R}$ & 14 & 16 & 26 & $3.75^{* *}$ & 14 & 0.47 \\
\hline BA 32 & L & -2 & 22 & 28 & $3.52^{* *}$ & 18 & 0.41 \\
\hline \multicolumn{8}{|c|}{ Midcycle/ovulation } \\
\hline \multicolumn{8}{|c|}{ (men $>$ women) } \\
\hline \multicolumn{8}{|c|}{ Cortical regions } \\
\hline \multicolumn{8}{|l|}{ ACG } \\
\hline BA 32 & $\mathrm{R}$ & 12 & 34 & 28 & 2.93 & 14 & 0.48 \\
\hline BA 24 & L & -4 & 32 & 8 & $3.12^{* *}$ & 27 & 0.32 \\
\hline BA 24 & L & -2 & 18 & 20 & 2.71 & 12 & 0.51 \\
\hline BA 24 & $\mathrm{R}$ & 4 & 32 & 8 & 2.63 & 6 & 0.66 \\
\hline BA 24 & $\mathrm{R}$ & 4 & 18 & 20 & 2.79 & 16 & 0.45 \\
\hline \multicolumn{8}{|l|}{$\mathrm{OFC}$} \\
\hline BA 10 & $\mathrm{R}$ & 34 & 58 & -4 & $4.06^{* *}$ & 83 & 0.09 \\
\hline BA 11 & $\mathrm{R}$ & 2 & 40 & -22 & 2.72 & 6 & 0.66 \\
\hline BA 47 & $\mathrm{R}$ & 24 & 18 & -18 & 2.98 & 9 & 0.58 \\
\hline BA 47 & $\mathrm{R}$ & 40 & 28 & -20 & $3.66^{* *}$ & 197 & 0.01 \\
\hline BA 47 & $\mathrm{~L}$ & -46 & 26 & -8 & $3.73^{* *}$ & 137 & 0.03 \\
\hline \multicolumn{8}{|l|}{ vmPFC } \\
\hline BA 10 & L & -4 & 42 & -20 & $3.25^{* *}$ & 33 & 0.19 \\
\hline BA 10 & $\mathrm{R}$ & 4 & 54 & -8 & $3.19^{* *}$ & 86 & 0.08 \\
\hline \multicolumn{8}{|l|}{ medPFC } \\
\hline BA 10 & $\mathrm{R}$ & 20 & 66 & 10 & $5.02^{* *}$ & 189 & 0.005 \\
\hline BA 10 & L & -4 & 64 & 20 & $3.62^{* *}$ & 56 & 0.09 \\
\hline \multicolumn{8}{|l|}{ medPFC/sFG } \\
\hline BA 10 & $\mathrm{R}$ & 34 & 58 & -4 & $4.06^{* *}$ & 66 & 0.07 \\
\hline \multicolumn{8}{|l|}{ Subcortical regions } \\
\hline Amygdala & L & -18 & -2 & -14 & $3.22^{* *}$ & 41 & 0.22 \\
\hline Hippocampus & $\mathrm{R}$ & 30 & -24 & -8 & $4.28^{* *}$ & 308 & 0.003 \\
\hline \multicolumn{8}{|l|}{ Hypothalamus } \\
\hline $\operatorname{VMN}^{b}$ & L & -4 & 2 & -6 & 2.48 & 3 & 0.77 \\
\hline \multicolumn{8}{|l|}{ Brainstem } \\
\hline PAG & & 2 & -30 & -2 & 2.96 & 60 & 0.14 \\
\hline
\end{tabular}

Voxelwise Z-score significance level is shown. ${ }^{* *} p \leq 0.001$, uncorrected for multiple comparisons within a hypothesized ROI; ROIs listed represent regions of significantly activated clusters within the a priori hypothesized ROI. BA Brodmann area; medPFC, medial prefrontal cortex; $\mathrm{sFG}$, superior frontal gyrus.

${ }^{a}$ FWE rate used for SVC: cluster-level significance level (FWE corrected within the search volume of interest).

${ }^{b} \mathrm{VMN}$ : derived from a $4 \mathrm{~mm}$ sphere around activation center; the region was identified by MNI coordinates and validated by neuroanatomist (N.M.). [Note: Left LHA, which was derived from a $4 \mathrm{~mm}$ sphere around activation center, had a significant Z-score at 2.47 (coordinates $-4,-2,-8$ ), but with only 1 voxel, and therefore was not included in the table above.]

In contrast, comparisons of negative-to-neutral-stimuli for men versus women during the late follicular/midcycle phase showed a greater number and extent of differential regions activated in men (Table 2). BOLD signal changes in men compared to women were greater in the anterior cingulate gyrus, OFC, medial and ventromedial PFC, medial PFC/superior frontal gyrus, amygdala, and hippocampus. Medial PFC, OFC, and hippocampus remained significant based on the more conservative small volume correction using the familywise error rate. Comparing LF/MC women to men, there were no significant signal intensity changes in the stress response circuitry.

Table 3 presents sex differences in tests of signal intensity changes in our set of functionally defined ROIs [chosen based on our previous significant findings in women (Goldstein et al., 2005) and findings presented here]. Figure 2 illustrates these differences comparing effect sizes of signal intensity changes in these regions in men versus EF women and men versus LF/MC women. As seen here, men significantly differed from EF women on bilat- 


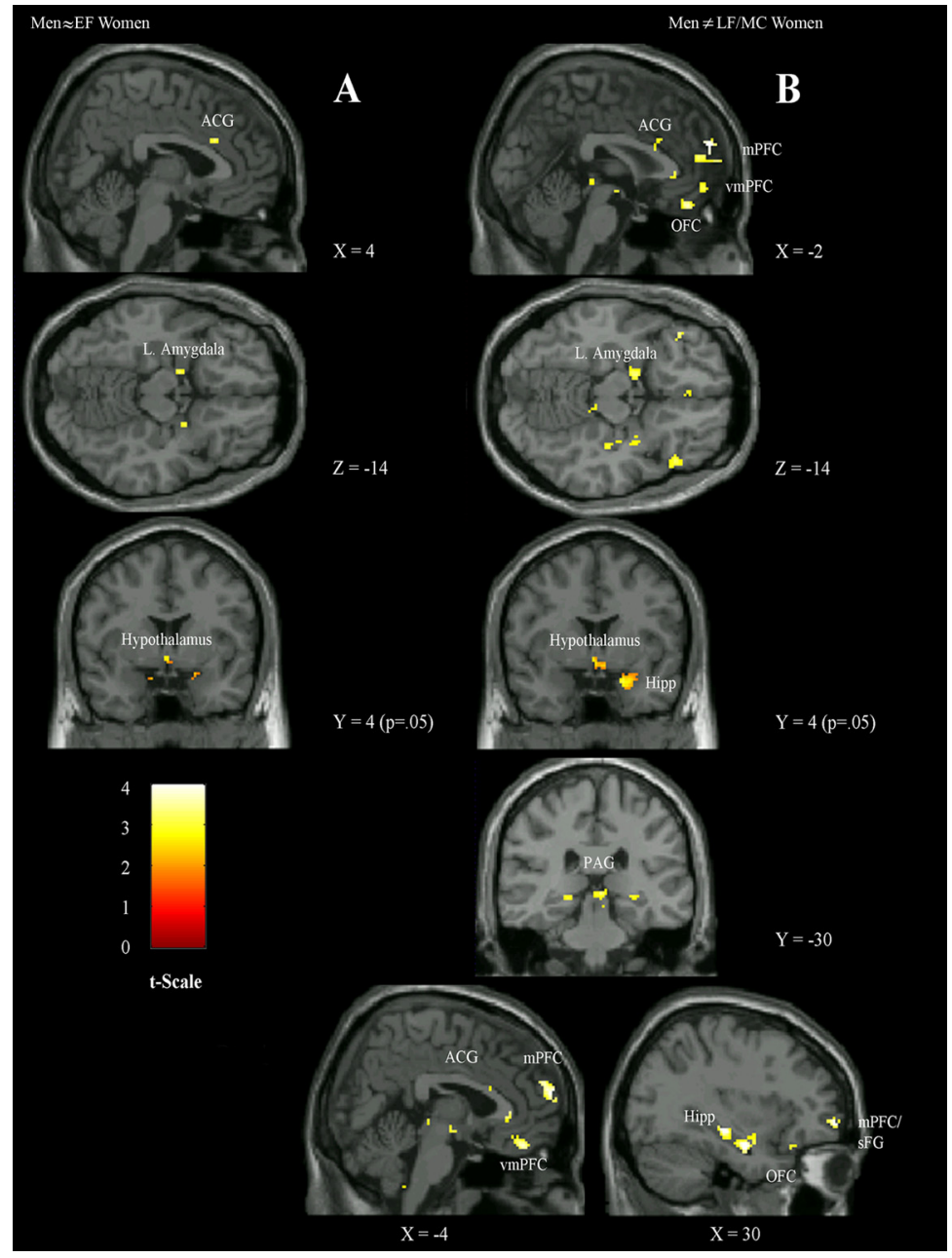

Figure 1. Significant sex differences in stress response regions comparing men versus women in early follicular $(\boldsymbol{A})$ and late follicular/midcycle menstrual (B) phases. sFG, Superior frontal gyrus; Hipp, hippocampus; Hypothalamus, lateral hypothalamic area and ventromedial nucleus of the hypothalamus. Activations of hypothesized regions of interest were derived from $8 \mathrm{~mm}$ sphere around activation center; hypothalamic areas were from $4 \mathrm{~mm}$ spheres. Activations in Figure 1 are selected from Table 3, representative of hypothesized regions that were significantly different between men and $\mathrm{EF}$ women and men and LF/MC women (Table 3, columns 6,7). For illustrative purposes alone, the peak activation in each activation cluster has an uncorrected $p<0.005$, except for the hypothalamus at $p<0.05$.

eral ACG, amygdala, and hypothalamus (LHA and VMN). Men significantly differed from LF/MC women on bilateral ACG and OFC, medial and ventromedial PFC, amygdala, hippocampus, LHA, VMN, and the brainstem. Findings were most pronounced (i.e., largest effect sizes) comparing cortical arousal regions, in particular medial and ventromedial PFC (with significant effect sizes of 0.60 and 0.61 ) (Table 3, column 7; Fig. 2).

The strongest evidence for sex differences is reflected in the formal testing of whether there were "different differences" in signal intensity comparisons between men versus EF women and men versus LC/MC women (Table 3, last column). These tests demonstrated significant effect size differences for bilateral OFC and medial and ventromedial PFC, right hippocampus and amygdala, and PAG (Table 3 ). The effect sizes were particularly large for medial and ventromedial PFC and OFC, critical brain regions implicated in the cortical control of arousal (Dougherty et al., 2004; Shin et al., 2004; Milad et al., 2007).

\section{Discussion}

In this study, we provide compelling evidence that sex differences in brain activity in the stress response circuitry are accounted for by women's hormonal status. We suggest that gonadal hormones play a role in the regulation of arousal via the impact of subcortical brain activity on the cortical control of arousal. This has significant implications for elucidating the neurobiological mechanism of the control of stress as well as understanding basic physiological differences in the male and female brain in response to stress.

We demonstrated that, in the face of stressful stimuli, subcortical arousal circuitry, i.e., hypothalamic nuclei and left amygdala, along with ACG, showed greater activation in men than women, regardless of women's cycle timing. That is, in comparing men to EF women, there was significantly greater activation in men of the left amygdala, hypothalamic nuclei (LHA and VMN), and bilateral ACG. All other stress response regions activated similarly in men compared with EF women. In contrast, compared to LF/MC women, men exhibited greater activation in ACG, OFC, medial PFC, left amygdala, hippocampus, LHA, VMN, and brainstem regions (e.g., PAG and midbrain). Thus, we demonstrated that stress response circuitry activated in the male brain is more similar to women in the EF menstrual phase compared with women during LF/MC. The strongest evidence for this is seen in Table 3 , in which we averaged signal intensity values in voxels across a priori defined anatomic regions in the stress response circuitry. These findings suggest that compared to men, there is an attenuation of stress response circuitry activation in women, particularly evident during midcycle, in subcortical arousal regions that couple with attenuation of regions implicated in the cortical control of arousal. In fact, the largest sex difference effect sizes were in the ventral and medial prefrontal cortices.

Given that EF (when estrogen and progesterone are low) is hormonally more similar to men compared with LF/MC (when estrogen is high), we would argue that it is likely estrogen or ratio of estrogen: progesterone that accounts for activation differences. A recent study of reward circuitry, which shares some brain regions with the stress response (e.g., amygdala, hypothalamus, OFC, and ACG), demonstrated regionspecific gonadal hormonal regulation, particularly by estrogen unopposed by progesterone (Dreher et al., 2007). Findings by Dreher et al. (2007) are not directly comparable, given that they tested women at different points in the menstrual cycle than our study and did not directly test for hormonal effects on sex differences. However, the Dreher et al. (2007) study does underscore the importance of hormonal regulation in related brain circuitry and the role of estrogen unopposed by progesterone (as would be similar to women during midcycle in our study).

Sex differences in negative affect were directly tested in two recent fMRI studies (Schienle et al., 2005; Caseras et al., 2007), 
Table 3. Signal change values in hypothesized stress response circuitry regions comparing negative to neutral stimuli in men versus women in early follicular and late follicular/midcycle periods

\begin{tabular}{|c|c|c|c|c|c|c|c|}
\hline Hypothesized regions $^{a}$ & R/L Hem & Men & EF Women & LF/MC Women & Difference in $\mathrm{ES}^{b}$ : men versus $\mathrm{EF}$ & Difference in $\mathrm{ES}^{b}$ : men versus $\mathrm{LF} / \mathrm{MC}$ & Test of different differences in ESC \\
\hline \multicolumn{8}{|l|}{ Cortical } \\
\hline \multicolumn{8}{|l|}{ ACG } \\
\hline \multirow[t]{2}{*}{ BA 24, 32} & L & 0.20 & -0.08 & -0.15 & $0.28^{*}$ & $0.35^{* *}$ & -0.07 \\
\hline & $\mathrm{R}$ & 0.05 & -0.15 & -0.16 & $0.20^{*}$ & $0.21^{*}$ & -0.01 \\
\hline \multicolumn{8}{|l|}{$\mathrm{OFC}$} \\
\hline \multirow[t]{2}{*}{ BA $10,11,47$} & L & 0.39 & 0.15 & -0.20 & 0.24 & $0.59^{* *}$ & $-0.35^{* *}$ \\
\hline & R & 0.29 & 0.10 & -0.19 & 0.19 & $0.49^{* *}$ & $-0.30^{* *}$ \\
\hline \multicolumn{8}{|l|}{ medPFC } \\
\hline BA 10, 11 & $\mathrm{R}$ & 0.24 & 0.26 & -0.36 & -0.02 & $0.60^{* *}$ & $-0.62^{* * *}$ \\
\hline \multirow[t]{2}{*}{ vmPFC } & L & 0.31 & 0.07 & -0.29 & -0.024 & $0.61^{* *}$ & $-0.37^{* * *}$ \\
\hline & $\mathrm{R}$ & 0.52 & 0.52 & -0.02 & 0.00 & $0.54^{*}$ & $-0.54^{* * *}$ \\
\hline \multicolumn{8}{|l|}{ Subcortical } \\
\hline \multirow[t]{2}{*}{ Amygdala } & L & 0.36 & 0.09 & 0.01 & $0.27^{*}$ & $0.35^{*}$ & -0.08 \\
\hline & $\mathrm{R}$ & 0.06 & 0.05 & -0.09 & 0.01 & 0.15 & $-0.14^{*}$ \\
\hline Hippocampus & R & 0.25 & 0.12 & -0.05 & 0.13 & $0.30^{* * *}$ & $-0.18^{* *}$ \\
\hline \multicolumn{8}{|l|}{ Hypothalamus } \\
\hline LHA & L & 0.58 & 0.15 & 0.17 & $0.43^{*}$ & $0.41^{*}$ & -0.02 \\
\hline VMN & L & 0.43 & 0.14 & 0.12 & $0.29^{*}$ & $0.31^{*}$ & -0.03 \\
\hline \multicolumn{8}{|l|}{ Brainstem } \\
\hline Medulla & & 0.10 & 0.04 & -0.03 & 0.06 & 0.13 & -0.07 \\
\hline Midbrain & & 0.19 & 0.02 & -0.06 & 0.18 & $0.25^{*}$ & -0.08 \\
\hline PAG & & 0.48 & 0.48 & 0.08 & 0.01 & $0.40^{*}$ & $-0.40^{*}$ \\
\hline Pons & & 0.15 & 0.07 & -0.07 & 0.08 & $0.22^{+}$ & -0.15 \\
\hline
\end{tabular}

Significance levels: ${ }^{* * *} p \leq 0.001 ;{ }^{* *} p \leq 0.01 ;{ }^{*} p \leq 0.05 ;{ }^{+} p \leq 0.10$; two-tailed. medPFC, Medial prefrontal cortex.

${ }^{a}$ Hypothesized region of interest activations were derived from an $8 \mathrm{~mm}$ sphere around activation center and hypothalamic areas from a $4 \mathrm{~mm}$ sphere.

${ }^{b} \mathrm{ESS}=$ standard deviations calculated as follows: differences between negative versus neutral signal intensity changes in men versus EF women and men versus LF/MC women; differences are divided by the SD of signal intensity value of the whole sample.

'The differences between two different signal intensity values (i.e. men vs EF women and men vs LF/MC women) was tested formally using PROC MIXED in SAS. This procedure fits mixed linear models and handles repeated measurements in order to take into account the variance of the two different measurements (EF and LF/MC) for each woman in the current study.

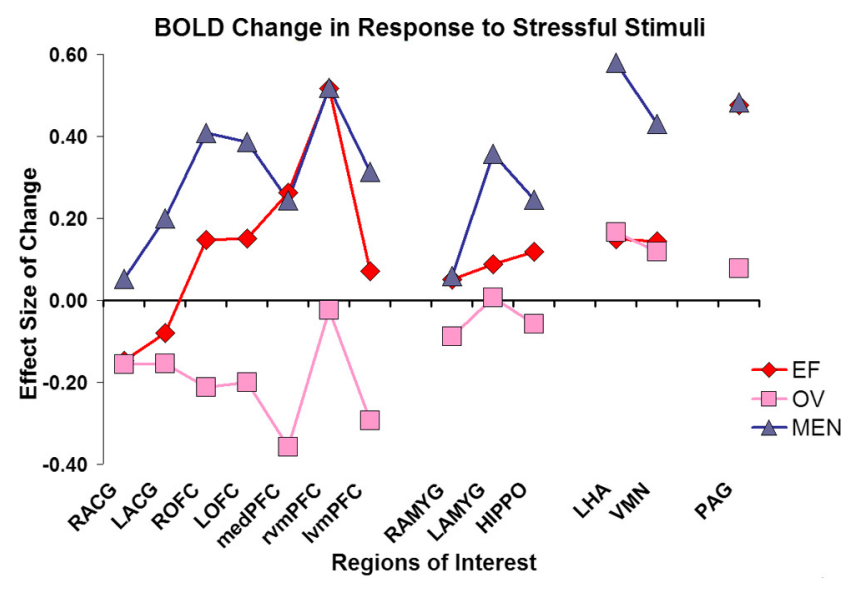

Figure 2. Effect sizes of BOLD signal intensity changes in response to stressful stimuli in men, women during early follicular, and women during late follicular/midcycle. This is a graphic representation of effect sizes in signal intensity changes (data reported in Table 3, columns 6,7; see table for relevant statistics) for our hypothesized regions of interest in the stress response circuitry. ESs = SDs calculated as follows: differences between negative versus neutral signal intensity changes in men versus EF women and men versus LF/MC women; differences are divided by SD of signal intensity value of the whole sample. The graph illustrates the differences between men (in blue) and women during early follicular (in red) and women during midcycle (in pink) phase of the menstrual cycle. The abbreviations represent the following regions of interest (L, left hemisphere; $R$, right hemisphere): medPFC, medial prefrontal cortex; HIPPO, hippocampus.

suggesting greater activation of amygdala in men (Schienle et al., 2005) and left ventrolateral PFC in women (Caseras et al., 2007). However, gender groups were only matched on age and handedness, and stimulus and contrast images were not matched on faces and scenes, producing potential confounding influ- ences on sex differences in brain activity. Further, lack of control for menstrual status may have attenuated sex differences in both studies. Finally, subjective valence and arousal ratings of images differed in men and women (Schienle et al., 2005; Caseras et al., 2007) and accounted for findings by Caseras et al. (2007), in contrast to our study demonstrating sex differences in brain activations given similar subjective ratings of arousal and valence. Our findings importantly suggest sex differences in the brain's response to stress even in the context of similar clinical state and subjective behavioral ratings, suggesting a role for hormones in regulating homeostasis in the brain in response to stress.

Previous animal studies demonstrated that testosterone $(\mathrm{T})$ regulated norepinephrine (NE) levels during prenatal development and differentially by sex (Stewart and Rajabi, 1994), suggesting an association between HPG and HPA circuitry. That is, inhibiting aromatase (the enzyme that converts $\mathrm{T}$ to estrogen) during development produced a rise in NE in anterior frontal, insular, and cingulate cortices (Stewart and Rajabi, 1994). This is consistent with our findings in men compared with LF/MC women, in which we demonstrated a large sex difference in the LHA, a key region in the noradrenergic pathway (Luiten et al., 1987), coupled with regions implicated in the cortical arousal circuitry (ACG, OFC, and vmPFC).

LHA has a primary role in arousal and is connected with cortical circuitry implicated in the inhibitory control of arousal and motivational shifts in behavior. Thus it contributes to maintaining homeostasis in the face of the fight/flight arousal response (Swanson, 1987). Some of the densest connections of the LHA are with $\mathrm{mPFC}$ and OFC, demonstrated in rats (Luiten et al., 1987) and cats (Room and Groenewegen, 1986), and it is part of the reticular system regulating the autonomic nervous system (ANS) 
and somatomotor circuitry (Swanson, 1987). In fact in our study, BOLD signal intensity values in activation of LHA correlated significantly and positively with those of vmPFC and OFC $[r=$ 0.55 and 0.66 ( $p \leq 0.005)$, respectively].

LHA is also a primary region in the noradrenergic pathway with the PVN (Luiten et al., 1987) (hypothalamic nuclei with the greatest concentration of corticotropin releasing hormone), thus providing an anatomical basis of prefrontal control of the HPA axis. In fact, LHA and VMN were qualitatively different in the male and female response circuitry, i.e., LHA and VMN were significantly activated in men, and, compared with women, not dependent on cycle phase. Sex differences in hypothalamic activations may be one reason for sex differences in cortisol levels in response to stress, regulated in part by the PVN in releasing adrenocorticotropin hormone (Schaeffer and Baum, 1984), which interacts with LHA and VMN.

Increases in hypothalamic activations in men may have necessitated compensatory increases in prefrontal circuitry to provide cortical control and homeostasis, in part reflected in the positive correlations of LHA with vmPFC and OFC (noted above). In fact, BOLD signal intensity values in activations of vmPFC and OFC were also significantly and positively correlated with the amygdala $[r=0.61$ and 0.65 ( $p \leq 0.001)$, respectively $]$ and hippocampus $[r=0.72$ and $0.65(p \leq 0.005)$, respectively]. Previous literature has argued that BOLD signal is mainly associated with excitatory inputs to cells rather than their output spikes (Logothetis, 2008), and BOLD changes can be associated with increased inhibition depending on the region being inhibited and experimental conditions (Buzsáki et al., 2007; Logothetis, 2008). In our study, BOLD activity in subcortical regions was expected to represent both an early increase in excitatory inputs, signaling arousal, followed by an increase in cortical inhibitory inputs to inhibit arousal. This would result in an effect that was consistent with a net increase in BOLD activity (i.e., positive correlations between cortical and subcortical activity). Positive correlations were also found between vmPFC and hippocampus and amygdala during recall of fear (Milad et al., 2007), which was interpreted as consistent with the top-down inhibitory control of subcortical arousal by vmPFC (Milad et al., 2007). In fact, the associations of cortical arousal circuitry to regulation of subcortical arousal regions have been disrupted in disorders such as anxiety and major depression (Shin et al., 2001; Dougherty et al., 2004) and autism (Bachevalier and Loveland, 2006), for which stress response and fear circuitries have been implicated.

The modulatory role of cortical arousal circuitry fits with our previous findings (Goldstein et al., 2005) and others (Protopopescu et al., 2005) in women and with studies demonstrating an inhibitory role of estradiol on arousal circuitry (Best et al., 1992; Lindheim et al., 1994; Kirschbaum et al., 1996). Although some preclinical studies have demonstrated excitatory roles for estradiol (Woolley and McEwen, 1993; Segal and Murphy, 2001; Lund et al., 2005), this does not negate an inhibitory role for estradiol, which has been associated with estrogen receptor $\beta$ (Ostlund et al., 2003; Shansky et al., 2004; Bao et al., 2005; Lund et al., 2005) while the excitatory role may be associated with estrogen receptor $\alpha$ (Lund et al., 2006). These opposing effects of $\mathrm{ER}_{\alpha}$ and $\mathrm{ER}_{\beta}$ in response to stress have been demonstrated at the level of behavior (Lund et al., 2005) and the level of the anterior hypothalamus (Lund et al., 2006).

Thus, our findings present a potential mechanism for the regulation of the stress response by circulating hormones in women. We also showed that there is not a general effect of hormones on overall blood flow or brain activity, but hormonal effects are region specific. This makes sense given that these brain regions control the HPA and HPG axes, involve brainstem regions implicated in ANS function, and provide frontal cortical influence over autonomic and endocrine function (Price, 1999). They are also dense in sex steroid receptors (McEwen, 1981; Keverne, 1988; Ostlund et al., 2003; Bao et al., 2005; Lund et al., 2005), noradrenergic receptors (among other monoaminergic receptors), and vasopressin and oxytocin (Keverne, 1988; De Vries and alShamma, 1990; Feldman et al., 1995; Pacak et al., 1995; Tobet and Hanna, 1997; Price, 1999; McEwen, 2000; Swaab, 2004), thus underscoring their adrenal and ovarian functions.

In summary, findings in this study demonstrated that the male and female brain differs in brain activity in response to stressful stimuli, even given the same clinical or behavioral response. Hormonal changes in women during midcycle result in an attenuation of subcortical arousal coupled with attenuation in cortical arousal circuitry that differs from men. This may have important clinical implications for understanding sex differences in clinical disorders associated with stress response circuitry (McEwen, 2000) and high rates of comorbid endocrine disorders.

From an evolutionary point of view, it is important for the female during midcycle to have a heightened cortical capacity, unencumbered by excessive arousal, to optimally judge whether a potentially threatening stimulus, such as an approaching male, is an opportunity for successful mating or for fight or flight. Thus, females have been endowed with a natural hormonal capacity to regulate the stress response that differs from males. This mechanism may have been maladaptive or unnecessary from an evolutionary point of view for the male, who had primary responsibility for protection of the species, thus necessitating a constant fight-or-flight behavioral response. Historically, these complementary sex-specific social roles have been dynamic, and this may reflect sex-specific plasticity in these arousal circuitry neural systems. Thus, although there are sex differences in these neurobiologic systems, they may now support some of the same social functions.

\section{References}

Bachevalier J, Loveland KA (2006) The orbitofrontal-amygdala circuit and self-regulation of social-emotional behavior in autism. Neurosci Biobehav Rev 30:97-117.

Bao AM, Hestiantoro A, Van Someren EJ, Swaab DF, Zhou JN (2005) Colocalization of corticotropin-releasing hormone and oestrogen receptor- $\alpha$ in the paraventricular nucleus of the hypothalamus in mood disorders. Brain 128:1301-1313.

Best NR, Rees MP, Barlow DH, Cowen PJ (1992) Effect of estradiol implant on noradrenergic function and mood in menopausal subjects. Psychoneuroendocrinology 17:87-93.

Bradley MM, Codispoti M, Sabatinelli D, Lang PJ (2001) Emotion and motivation II: sex differences in picture processing. Emotion 1:300-319.

Buzsáki G, Kaila K, Raichle M (2007) Inhibition and brain work. Neuron 56:771-783.

Caseras X, Mataix-Cols D, An SK, Lawrence NS, Speckens A, Giampietro V, Brammer MJ, Phillips ML (2007) Sex differences in neural responses to disgusting visual stimuli: implications for disgust-related psychiatric disorders. Biol Psychiatry 62:464-471.

De Vries GJ, al-Shamma HA (1990) Sex differences in hormone sensitivity of vasopressin pathways in the rat brain. J Neurobiol 21:686-693.

Dougherty DD, Rauch SL, Deckersbach T, Marci C, Loh R, Shin LM, Alpert NM, Fischman AJ, Fava M (2004) Ventromedial prefrontal cortex and amygdala dysfunction during an anger induction positron emission tomography study in patients with major depressive disorder with anger attacks. Arch Gen Psychiatry 61:795-804.

Dreher JC, Schmidt PJ, Kohn P, Furman D, Rubinow D, Berman KF (2007) Menstrual cycle phase modulates reward-related neural function in women. Proc Natl Acad Sci U S A 104:2465-2470.

Feldman S, Conforti N, Weidenfeld J (1995) Limbic pathways and hypotha- 
lamic neurotransmitters mediating adrenocortical responses to neural stimuli. Neurosci Biobehav Rev 19:235-240.

Friston KJ, Worsley KJ, Frackowiak RS, Mazziotta JC, Evans AC (2004) Assessing the significance of focal activations using their spatial extent. Hum Brain Mapp 1:210-220.

Goldstein JM, Seidman LJ, Horton NJ, Makris N, Kennedy DN, Caviness VS Jr, Faraone SV, Tsuang MT (2001) Normal sexual dimorphism of the adult human brain assessed by in-vivo magnetic resonance imaging. Cereb Cortex 11:490-497.

Goldstein JM, Jerram M, Poldrack R, Ahern T, Kennedy DN, Seidman LJ, Makris N (2005) Hormonal cycle modulates arousal circuitry in women using functional magnetic resonance imaging. J Neurosci 25:9309-9316.

Harlow BL, Cohen LS, Otto MW, Spiegelman D, Cramer DW (1999) Prevalence and predictors of depressive symptoms in older premenopausal women: the Harvard study of moods and cycles. Arch Gen Psychiatry 56:418-424.

Keverne EB (1988) Central mechanisms underlying the neural and neuroendocrine determinants of maternal behaviour. Psychoneuroendocrinology 13:127-141.

Kirschbaum C, Platte P, Pirke KM, Hellhammer DH (1996) Adrenocortical activation following stressful exercise: further evidence for attenuated free cortisol responses in women using oral contraceptives. Stress Med $12: 137-143$

Lane RD, Reiman EM, Bradley MM, Lang PJ, Ahern GL, Davidson RJ, Schwartz GE (1997) Neuroanatomical correlates of pleasant and unpleasant emotion. Neuropsychologia 35:1437-1444.

Lang PJ, Greenwald MK, Bradley MM, Hamm AO (1993) Looking at pictures: affective, facial, visceral, and behavioral reactions. Psychopathology 30:261-273.

Lang PJ, Bradley MM, Fitzsimmons JR, Cuthbert BN, Scott JD, Moulder B, Nangia V (1998) Emotional arousal and activation of the visual cortex: an fMRI analysis. Psychophysiology 35:199-210.

Lang PJ, Bradley MM, Cuthbert BN (2008) International affective picture system (IAPS): affective ratings of pictures and instruction manual. Technical Report A-8. Gainesville, FL: University of Florida.

Lindheim SR, Legro RS, Morris RS, Wong IL, Tran DQ, Vijod MA, Stanczyk FZ, Lobo RA (1994) The effect of progestins on behavioral stress responses in postmenopausal women. J Soc Gynecol Investig 1:79-83.

Logothetis NK (2008) What we can do and cannot do with fMRI. Nature 453:869-878.

Luiten PG, ter Horst GJ, Steffens AB (1987) The hypothalamus, intrinsic connections and outflow pathways to the endocrine system in relation to the control of feeding and metabolism. Prog Neurobiol 28:1-54.

Lund TD, Rovis T, Chung WC, Handa RJ (2005) Novel actions of estrogen receptor-beta on anxiety-related behaviors. Endocrinology 146:797-807.

Lund TD, Hinds LR, Handa RJ (2006) The androgen $5 \alpha$-dihydrotestosterone and its metabolite $5 \alpha$-androstan- $3 \beta, 17 \beta$-diol inhibit the hypothalamo-pituitaryadrenal response to stress by acting through estrogen receptor $\beta$-expressing neurons in the hypothalamus. J Neurosci 26:1448-1456.

Maldjian JA, Laurienti PJ, Kraft RA, Burdette JH (2003) An automated method for neuroanatomic and cytoarchitecture atlas-based interrogation of fmri data sets. Neuroimage 19:1233-1239.

McClure EB, Monk CS, Nelson EE, Zarahn E, Leibenluft E, Bilder RM, Charney DS, Ernst M, Pine DS (2004) A developmental examination of gender differences in brain engagement during evaluation of threat. Biol Psychiatry 55:1047-1055.

McEwen BS (1981) Neural gonadal steroid actions. Science 211:1303-1311.

McEwen BS (2000) The neurobiology of stress: from serendipity to clinical relevance. Brain Res 886:172-189.
McEwen BS, Magarinos AM (1997) Stress effects on morphology and function of the hippocampus. Ann N Y Acad Sci 821:271-284.

Milad MR, Wright CI, Orr SP, Pitman RK, Quirk GJ, Rauch SL (2007) Recall of fear extinction in humans activates the ventromedial prefrontal cortex and hippocampus in concert. Biol Psychiatry 62:446-454.

Ostlund H, Keller E, Hurd YL (2003) Estrogen receptor gene expression in relation to neuropsychiatric disorders. Ann N Y Acad Sci 1007:54-63.

Pacak K, Palkovits M, Kopin IJ, Goldstein DS (1995) Stress-induced norepinephrine release in the hypothalamic paraventricular nucleus and pituitary-adrenocortical and sympathoadrenal activity: in vivo microdialysis studies. Front Neuroendocrinol 16:89-150.

Price JL (1999) Prefrontal cortical networks related to visceral function and mood. Ann N Y Acad Sci 877:383-396.

Protopopescu X, Pan H, Altemus M, Tuescher O, Polanecsky M, McEwen B, Silbersweig D, Stern E (2005) Orbitofrontal cortex activity related to emotional processing changes across the menstrual cycle. Proc Natl Acad Sci U S A 102:16060-16065.

Room P, Groenewegen HJ (1986) Connections of the parahippocampal cortex in the cat. II. Subcortical afferents. J Comp Neurol 251:451-473.

Schaeffer MA, Baum A (1984) Adrenal cortical response to stress at Three Mile Island. Psychosom Med 46:227-237.

Schienle A, Schäfer A, Stark R, Walter B, Vaitl D (2005) Gender differences in the processing of disgust- and fear-inducing pictures: an fMRI study. Neuroreport 16:277-280.

Segal M, Murphy D (2001) Estradiol induces formation of dendritic spines in hippocampal neurons: functional correlates. Horm Behav 40:156-159.

Shansky RM, Glavis-Bloom C, Lerman D, McRae P, Benson C, Miller K, Cosand L, Horvath TL, Arnsten AF (2004) Estrogen mediates sex differences in stress-induced prefrontal cortex dysfunction. Mol Psychiatry 9:531-538.

Shin LM, Whalen PJ, Pitman RK, Bush G, Macklin ML, Lasko NB, Orr SP, McInerney SC, Rauch SL (2001) An fMRI study of anterior cingulate function in posttraumatic stress disorder. Biol Psychiatry 50:932-942.

Shin LM, Orr SP, Carson MA, Rauch SL, Macklin ML, Lasko NB, Peters PM, Metzger LJ, Dougherty DD, Cannistraro PA, Alpert NM, Fischman AJ, Pitman RK (2004) Regional cerebral blood flow in the amygdala and medial prefrontal cortex during traumatic imagery in male and female Vietnam veterans with PTSD. Arch Gen Psychiatry 61:168-176.

Stewart J, Rajabi H (1994) Estradiol derived from testosterone in prenatal life affects the development of catecholamine systems in the frontal cortex in the male rat. Brain Res 646:157-160.

Swaab DF (2004) The human hypothalamus. Basic and clinical aspects. Part II: Neuropathology of the hypothalamus and adjacent brain structures. In: Handbook of clinical neurology (Aminoff MJ, Boller F, Swaab DF, eds), p 596. Amsterdam: Elsevier.

Swanson LW (1987) The hypothalamus. In: Handbook of chemical neuroanatomy, Vol 5, Integrated systems of the CNS, Part I (Hökfelt T, Björklund A, Swanson LW, eds), pp 1-124. Amsterdam: Elsevier.

Tobet SA, Hanna IK (1997) Ontogeny of sex differences in the mammalian hypothalamus and preoptic area. Cell Mol Neurobiol 17:565-601.

Woolley CS, McEwen BS (1993) Roles of estradiol and progesterone in regulation of hippocampal dendritic spine density during the estrous cycle in the rat. J Comp Neurol 336:293-306.

Wrase J, Klein S, Gruesser SM, Hermann D, Flor H, Mann K, Braus DF, Heinz A (2003) Gender differences in the processing of standardized emotional visual stimuli in humans: A functional magnetic resonance imaging study. Neurosci Lett 348:41-45. 